\title{
Lock-in Thermography for Process Integrated Non-Destructive Evaluation of Carbon Fibre Reinforced Aircraft Structures
}

\author{
by T. Ullmann*, R. Aoki* and T. Schmidt** \\ * Institute of Structures and Design, German Aerospace Center DLR, Pfaffenwaldring 38-40, 70569 Stuttgart, \\ Germany, Thomas.Ullmann@dlr.de, R.Aoki@dlr.de \\ ** Institute of Structures and Design, German Aerospace Center DLR, Alter Postweg 101, 86159 Augsburg, \\ Germany, T.Schmidt@dlr.de
}

\begin{abstract}
Reliable process integrated quality assurance for the manufacturing of large-scale CFRP aircraft structures, such as fuselage shells, cargo doors or components of a central wing box, belongs to one of the most challenging objectives for nondestructive testing. Beside the well known key criteria like non-contact measurement and imaging options as well as the maximum failure detection resolution and defect selectivity even more aspects have to be considered: the penetration depth into the component's material, the scanning and measurement speed, the coverage area as well as the accessibility of the structure (inspection condition with single side or two side accessibility). The probably most significant requirements for process integrated non-destructive inspection are the measurement time and data evaluation. Therefore the coverage area and the measurement speed are quite important. For example common ultrasound inspection screens the component's surface line by line until the whole area is scanned. This requires quite a lot of time and as soon as the component's shape includes curved or ribbed areas, it becomes difficult to receive proper signals of the material's interior structure. In contrast, the main benefit of optical inspection methods is the coverage of relatively large areas within one exposure time but on the other hand only features which are located near the component's surface can be detected.

However, lock-in thermography seems to be a good compromise of all these boundary conditions as it is monitoring the time dependent change of thermal waves that are emitted by the structure component. Depending on the excitation frequency of the induced thermal waves, it is possible to collect information of the material's microstructural features from different depths and to cover relatively large areas within one measurement cycle. A single side accessibility of the structure component is absolutely sufficient and measurement time as well as data acquisition and interpretation can be performed within the order of minutes. Detecting and localizing material defects or significant microstructural changes is relatively easy since changes in the material's thermal diffusivity are clearly distinguishable in the visual data mapping of the component's surface. As previous work has shown, even a proceeding resin front of an infiltration process can be detected and visually displayed. Focusing the benefits and potentials it is shown that lock-in thermography is a valuable non-destructive inspection tool that can be used as part of a process integrated quality assurance system for CFRP structure component manufacturing.
\end{abstract}

\section{Introduction}

Due to upcoming requirements of reduced weight and significant savings in fuel consumption for future aircraft models, lightweight composite materials such as carbon fibre reinforced polymers (CFRP) are playing a more and more important role in new aircraft design concepts. With the models A350 XWB and B787, the big aircraft manufacturers Airbus and Boeing are currently developing a new generation of so called black airframes which are mainly composed of carbon fibre reinforced composites. The use of such a new material class does not only mean to exchange structural parts but to revise the whole structure design, component manufacturing and joining techniques as well as the material's machining procedures and quality assurance concept. Until now non-destructive testing has mainly been carried out on finished parts at the end of the manufacturing process. However, in order to reduce manufacturing costs and at the same time ensure high quality standards, it is inevitable to establish an automated monitoring system which is integrated into the production process chain. For example a CFRP pre-form structure which shows anomalies in the fibre layup after draping, will be removed from the process chain immediately in order to avoid the following process steps and therefore to minimize the manufacturing costs. For the separated part it then has to be decided whether it will be finally rejected or undergoes more detailed nondestructive inspection procedures.

Besides choosing appropriate non-destructive test methods for several manufacturing steps, it is also very important to provide an effective information management system with quick and full automated data evaluation and data storage. The analysis methods used in a process chain are carried out by complex measuring head systems, which are mounted on 
assembly robots and carry out fully automated test routines on the component. These must be adapted to the shape of the component and used on a large-scale in order to be able to inspect and assess the material properties of several square metres of large structure components in the shortest possible time.

\section{CFRP Component Design}

Fibre reinforced materials are layered materials with strongly anisotropic properties. The thermal expansion rigidity and strength of the materials differ greatly depending on the orientation of the fibres. When CFRP components are built and integrated into large structural components, fibre orientation plays a decisive role. An aircraft fuselage shell can be assembled from complete core-wound CFRP tubes (Boeing), or from several large CFRP shell segments (Airbus). In each case, the carbon fibres run along the surface of the fuselage body in order to make optimal use of their exceptional strength. The number of seams and joints has to be kept to a minimum, as these - in the same way as openings for windows, maintenance ports and cargo hold hatches - represent weaknesses in the structure. Unlike light metal fabrications, the direction-dependent material properties of the CFRP material determine the geometry of the components and the structural design. Figure 1 shows a detail view on a CFRP fuselage shell of Boeing's B787 with integral stringers and mounted stiffener elements.

The joining together of two fuselage segments presents a particular challenge, as the high dimensional stability of CFRP structural components allows a tolerance of only a few microns. Fuselage shells or segments, which are manufactured by different supplier plants that are located several hundreds of kilometres from each other, have to be joined together with an extremely high fit-accuracy during the final assembly process. As CFRP components cannot be welded together like light metal structures, the only possible joining techniques are gluing or riveting. For the construction of an aircraft, several hundred thousand metal rivets would be required. This additional mass would significantly reduce the achievable weight reduction. It would be a far better idea to glue the CFRP segments together. However, the adhesive bonding technique is a very demanding process, and has so far only been mastered to a limited extent. The developing of an appropriate verification technique, which can be used to check the characteristics of the bond, such as the surface coverage, the degree of hardening of the adhesive and its adherence to the surface of the components, is exceptionally difficult.

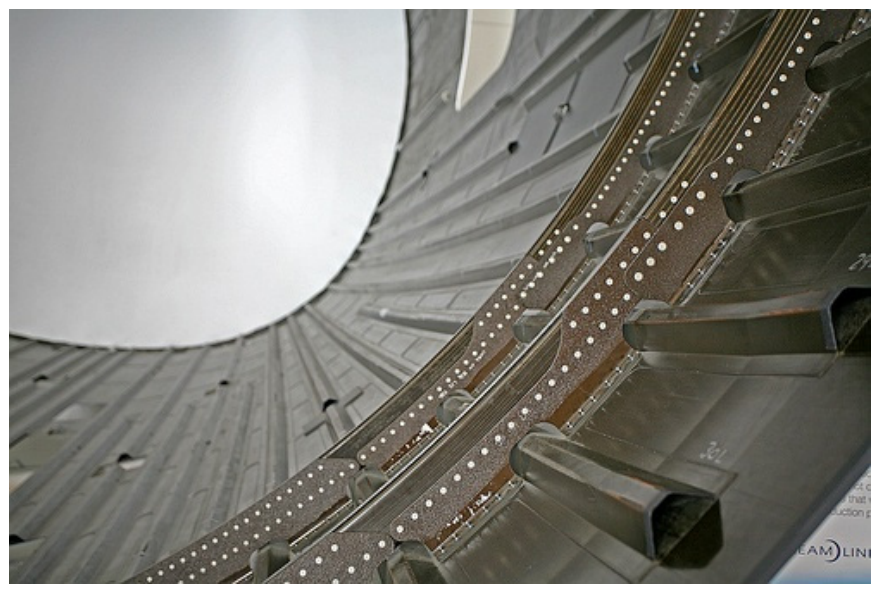

Fig. 1. Detail view of a CFRP fuselage section of Boeing's Dreamliner (Christopher Boffoli)

\section{In-line Quality Assurance}

At the DLR Center for Lightweight Production Technology (ZLP) in Stade and Augsburg, the expertise required for the cost-efficient, automated manufacturing and processing of large series of CFRP components is being acquired. In addition, a strategy for in-line quality assurance is being developed. This enables the monitoring and evaluation of individual processes during and between the different production stages. This also means that a non-destructive testing method to fully automatically monitor compliance with previously defined manufacturing tolerances based on particular properties, and that any deviations can be recognized and assessed by the analytical software. For semi-finished products and components with 
properties that indicate defective structures, this can mean that they are immediately removed from the production chain in order to prevent any further expensive and unnecessary manufacturing steps. The direct benefits of a quality assurance system that is integrated into the production process can only be fully assessed when considering the costs of performing the additional testing alongside the enormous savings as a result of the removal of defective parts from the production line in an early stage. The cost effective and efficient implementation of the non-destructive testing method in the individual processing stages requires fully automated and synchronized data acquisition and assessment that do not have substantial impact on the production process or even cause the interruption of the production process for a longer period [1].

Figure 2 shows a concept study of a multi-functional facility for component manufacturing that will be built up at the DLR Center for Lightweight Production Technology in Augsburg. Some of the robotic arms are supposed to carry the CFRP components and bring them in the right position for further manufacturing. Others are carrying machining tools and measurement heads, so called "end-effectors", for the following working steps and purposes of process monitoring. This facility will be capable to process components of several metres length.

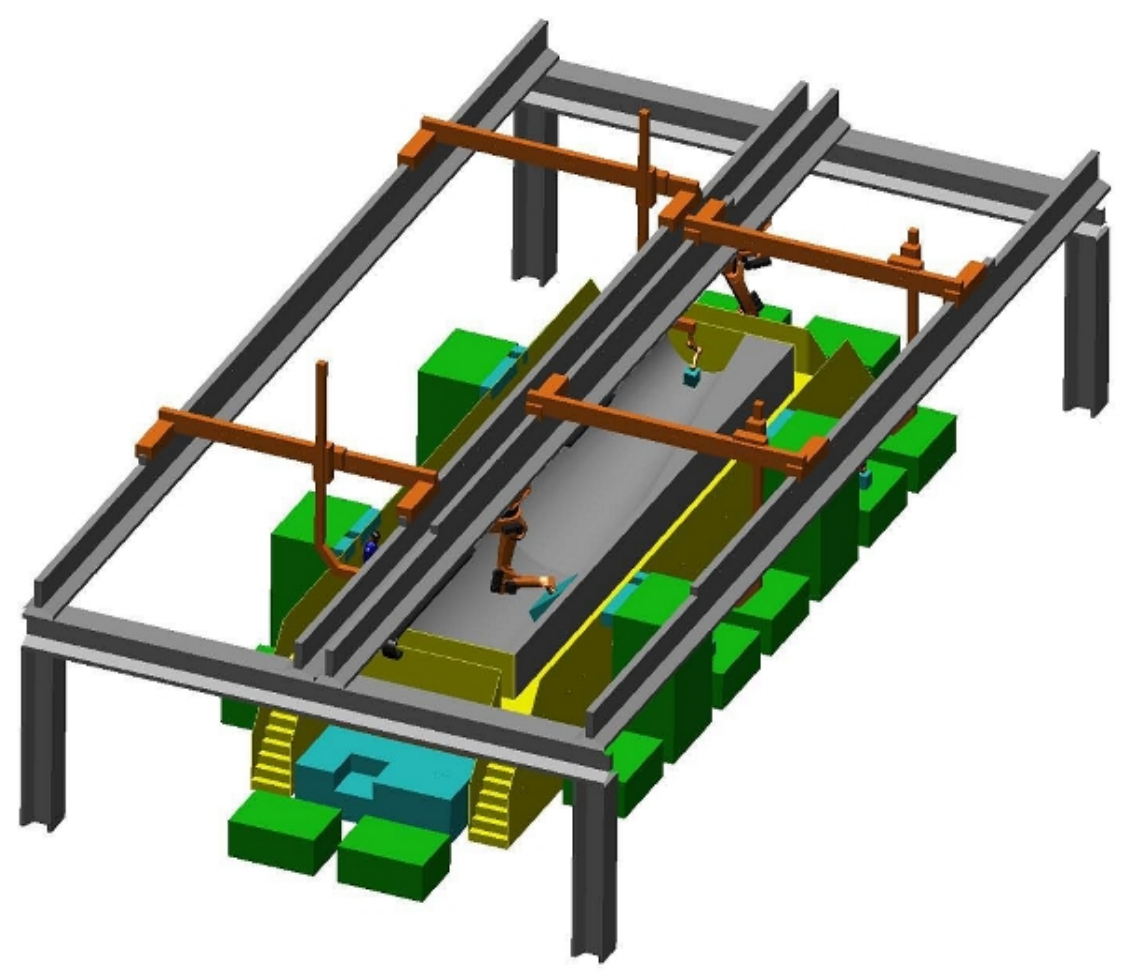

Fig. 2. Multi-functional manufacturing cell for CFRP structures (KUKA System)

\section{Lock-in Thermography for Process Integrated Failure Analysis}

\subsection{Lock-in Thermography}

The principle of lock-in thermography is based on the idea that temperature modulation induced from outside the surface of the component propagates as a "thermal wave" inside the material. As this wave undergoes reflections at boundaries like all other waves, there will be numerous reflecting thermal waves coming from the component's rear side and all internal boundaries (e.g. glued interfaces). As a consequence, the temperature modulation at the component's exposed surface is modified by thermal waves coming out from the inside of the structure. A sensitive indicator of the responding thermal wave is the phase angle between energy deposition and local thermal response. The resulting surface temperature field is monitored during the modulated illumination with a thermography camera that takes a sequence of infrared images. $\mathrm{A}$ 
Fourier analysis performed at each pixel of the sequence of infrared images provides information of the magnitude and phase of the local response wave. These two parameters may be used to illustrate the relevant information in a different kind of imaging. The magnitude image is affected by inhomogeneities of optical surface absorption, infrared emission and distribution of optical illumination. Nevertheless, when the evaluation is performed for each pixel of the image sequence, all these physical effects will be eliminated. Hence, the so called phase image does not show any of these disturbing effects at all. For curved aerospace structures it is often difficult to maintain a homogeneous optical illumination but with a temperature modulation in a sinusoidal way, this evaluation is particularly simple because averaging procedures reduce the about 1000 initial thermography images to only 4 which are by 90 degrees out of phase giving the phase image $\varphi$ according to

$$
\varphi=\arctan \frac{S_{3}-S_{1}}{S_{4}-S_{2}}
$$

where $S_{1}$ to $S_{4}$ denote the 4 images [2]. A sinusoidal thermal modulation can be achieved by electronic control of $4-8$ halogen lamps which are positioned in at least 2 groups of different orientation to the structure component. Figure 3 shows a typical test assembly as used at the NDE labs of DLR. The thermographic camera that is used here is a JADE III long wave infrared camera from CEDIP (spectral sensitivity: $7.7-9.3 \mu \mathrm{m}$; thermal sensitivity: $35 \mathrm{mK}$ ). The thermal energy is applied by 4 halogen lamps of $1200 \mathrm{~W}$ each [3]. According to the test assembly that was used for inspecting the aircraft rudder flap in Figure 3, the corresponding phase image is shown in Figure 4 below. Beside some of the rivets near the edge, there are a number of internal reinforcement elements that are clearly visible. As indicated by the scale, the colours represent the time displacement (phase shift) of the responding thermal waves from the inner material features of the CFRP structure. The phase image is a result of locally different thermal diffusivity in the rudder flap's structure.

According to the thermal diffusion length $\mu$ it is possible to obtain information about the geometry and location in depth of any defect in the surface region. The thermal diffusion length is given by:

$$
\mu=\sqrt{\frac{2 \lambda}{\omega \cdot \rho \cdot c_{p}}} \quad \Rightarrow \quad \mu=\sqrt{\frac{2 \alpha}{\omega}}
$$

Where the angular velocity of modulation is defined as $\omega=2 \pi f\left[\mathrm{~s}^{-1}\right]$, the thermal conductivity is $\lambda\left[\mathrm{W} \cdot \mathrm{m}^{-1}\right]$, material density is $\rho\left[\mathrm{kg} \cdot \mathrm{m}^{-3}\right]$, the specific heat capacity is $c_{p}\left[\mathrm{~J} \cdot \mathrm{kg}^{-1} \cdot \mathrm{K}^{-1}\right]$ and $\alpha$ denotes thermal diffusivity. The signal magnitude is affected by boundaries in a depth that is less than $\mu$ while signal phase still responds about twice this depth $[4,5]$.

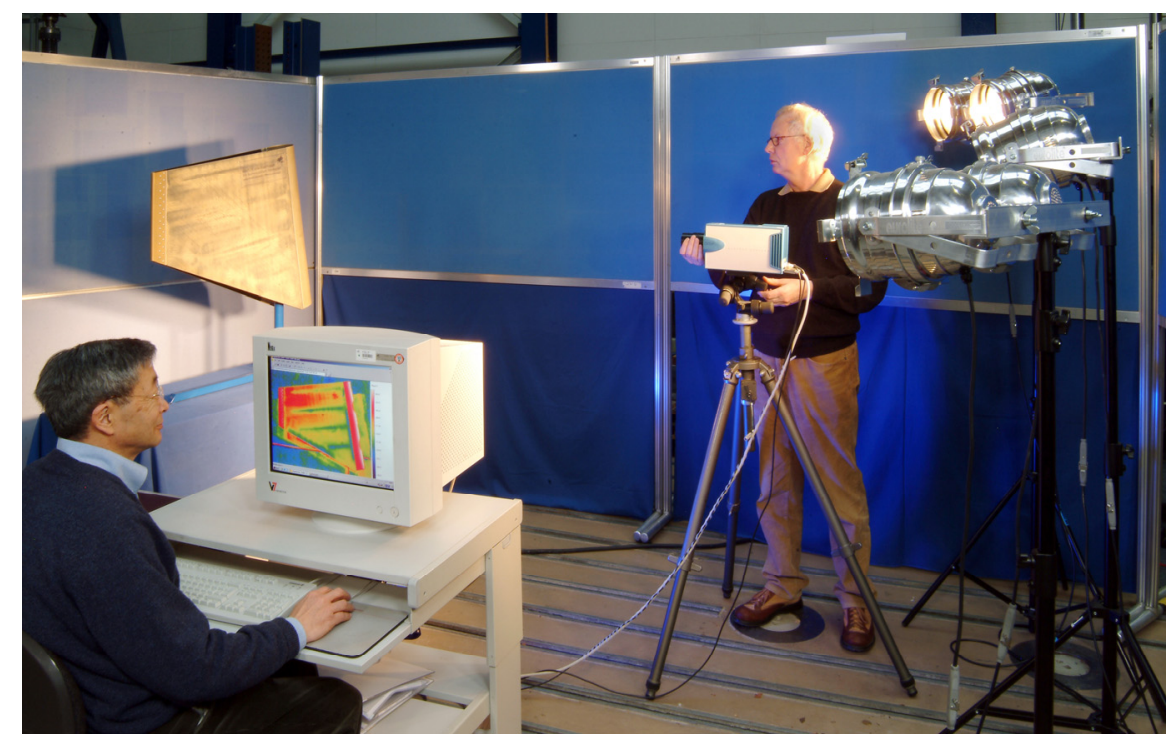

Fig. 3. Lock-in thermography analysis of a section of an aircraft rudder flap 


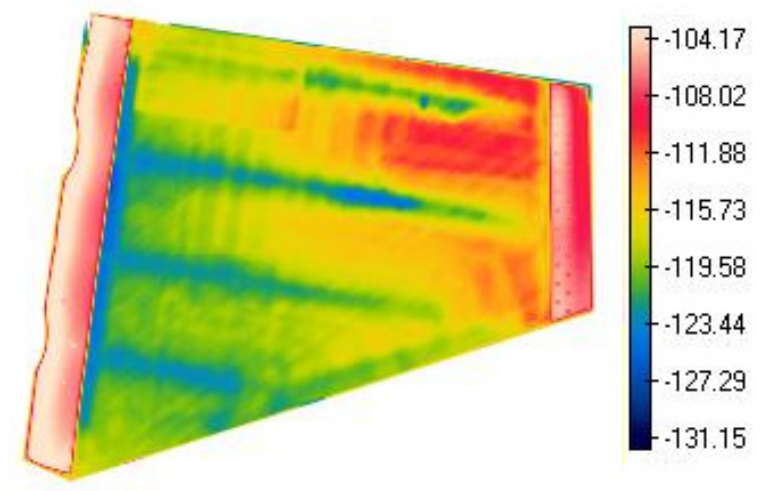

Fig. 4. Aircraft rudder flap with internal reinforcement elements and structures

Lock-in thermography analysis is a valuable test method which enables widespread scans on structure components in order to detect flaws and interfaces (e.g. glued interfaces) that are located near the material's surface. The maximum exposable surface area may be in the range of approximately $1 \mathrm{~m}^{2}$ and the standard measurement time is varying in the range of a few minutes. Furthermore as an optically excited measurement technique, lock-in thermography does not need any coupling media and a single side accessibility of the structure component is absolutely sufficient as long as the wall thickness of the material is not exceeding a few millimetres. Depending to the excitation frequency, structural features and material defects within $5-6 \mathrm{~mm}$ below the surface may be detected. The phase image shown in Figure 4 was obtained with an excitation frequency of $0.01 \mathrm{~Hz}$ within a total measurement time of $200 \mathrm{~s}$. As indicated by equation (2), phase images taken with lower excitation frequencies $f$ show details from increasing material depth. This is clearly demonstrated by the image comparison in Figure 5. These gray-scale phase images are the results of measurements with different excitation frequencies. While the left phase image from the measurement with the lowest penetration depth $(0.3 \mathrm{~Hz})$ shows more or less the arrangement of the carbon fibres, the right image, taken with the lowest excitation frequency $(0.01 \mathrm{~Hz})$, offers much more details from the interfaces with the reinforcement elements inside the structure. The right gray-scale image is the corresponding image to the one shown in Figure 4.
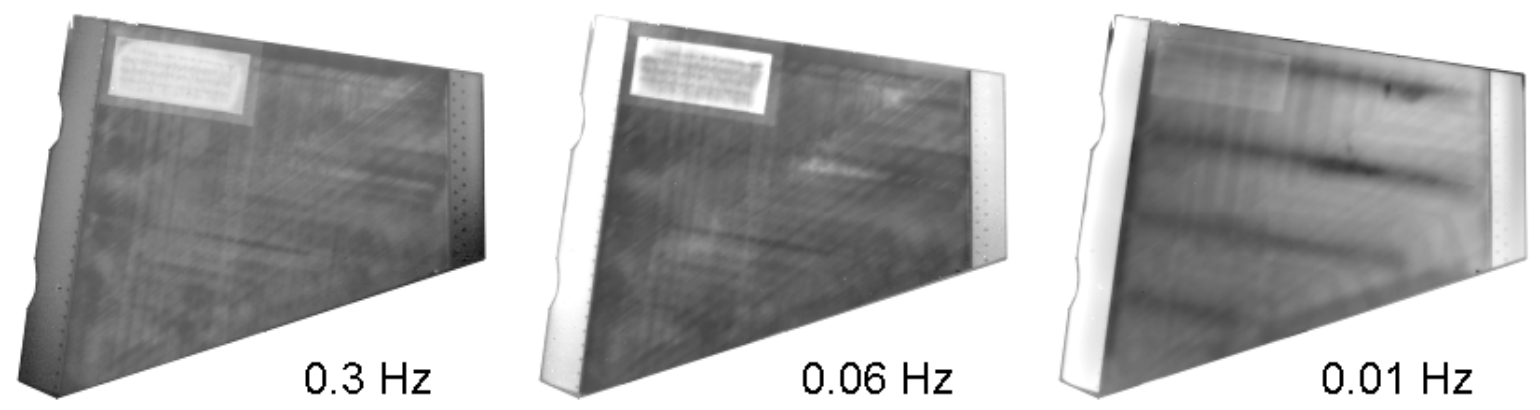

Fig. 5. Gray-scale phase images of lock-in thermography measurements at different excitation frequencies

Due to limited detail resolution and penetration depth the lock-in thermography technique does not solve any problem in terms of material defect detection. However, resuming all boundary conditions that have to be faced when inspecting large-scale CFRP components, it seems to be a valuable non-destructive measurement method for an automated and process integrated quality assurance system. Lock-in thermography enables a large-scale inspection of structural components, independent of their geometry. Typical features that might be monitored with lock-in thermography are regions of increased porosity, local delaminations or detached gluing layers. Furthermore, regarding the process monitoring of semifinished parts, this technique may be also used to control the appropriate placement of the carbon fibres or detecting missing rovings within a multi-axial fabric. Especially for the infiltration process monitoring, lock-in thermography enables the 
recognition of a locally inhomogeneous resin distribution that may not be visible from the surface but is capable to deteriorate mechanical strength and might initiate defects that could cause significant material damage as soon as the components will be in service later on.

As mentioned before, an automated operation of a lock-in thermography device would be carried out with a complex measurement head mounted on an assembly robot system just as shown in Figure 6 below. The robot system would be responsible for the right positioning of the thermography camera and the heating lamps with high accuracy. A large CFRP component of several metres in length and diameter would have to be monitored by different measurement cycles within a few minutes. The resulting phase images would be matched together like a puzzle. In the mean time each anomaly has to be localized with an accuracy of hundredths of millimetres and will then be compared to reference data from typical (possible) material defects formerly obtained by exemplary measurements with other non-destructive techniques with by far higher resolution (e.g. computed tomography). The decisive step is the automated decision by the analysis software whether a signal is interpreted as a nominal feature of the material's microstructure or a hint for a defect. The goal is to establish selflearning interpretation software that is capable to increase reliability of defect detection significantly with the amount of analysis cycles performed and the growing availability of reference data from other test methods with higher resolution.

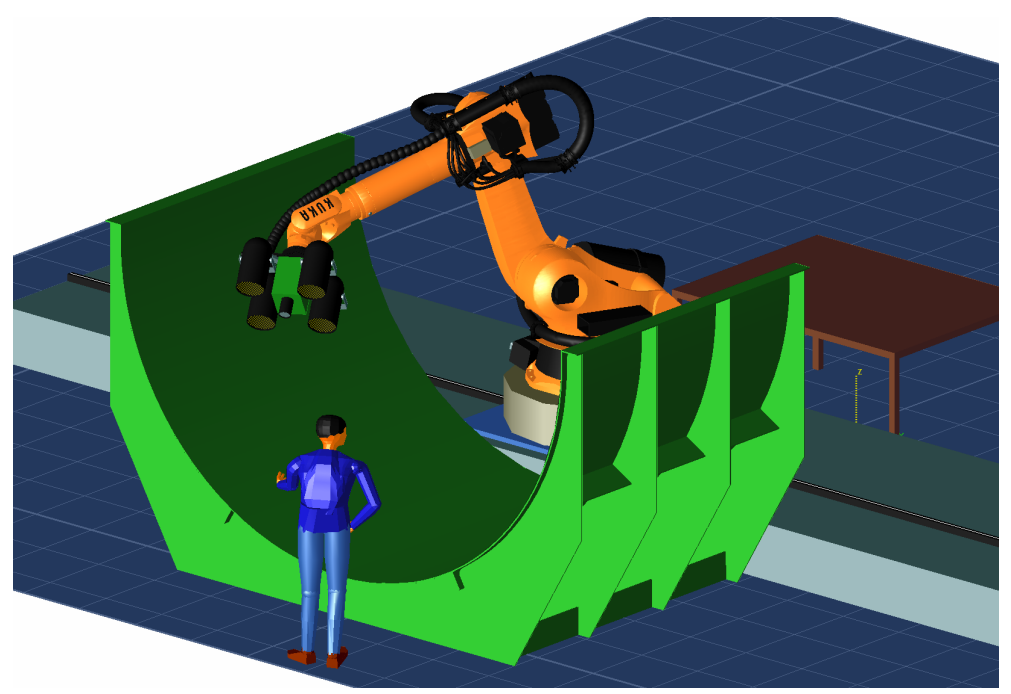

Fig. 6. Large area lock-in thermography of a measuring head which is mounted on a robot system

\subsection{Failure Analysis}

Some examples are demonstrating the potentials of the lock-in thermography measurement system. As previous work has shown, it is even possible to detect the proceeding resin front of an infiltration process [6,7]. As illustrated in Figure 7 , the region of dry fibre arrangement can be clearly distinguished from the impregnated area. Even the main orientations of the fibre rovings are clearly visible. Due to the sudden change of temperature diffusivity, the proceeding resin front is also visible. The colours of the images in Figure 7 are indicating the phase shift not the temperature distribution. According to Chatzgeorgiou, it is also possible to monitor the dynamic process of the proceeding resin front itself when using and comparing measurement series of adequate excitation frequencies [5]. However, the main obstacle so far is the problem of temperature dependence of the infiltration process. As soon as the component is placed in a furnace, it is not possible to apply the modulation of thermal waves and therefore performing a lock-in measurement at elevated temperatures of several hundred ${ }^{\circ} \mathrm{C}$ so far. Nevertheless, it is easily possible to detect inhomogeneities in resin distribution right after the infiltration process.

A CFRP plate of approximately $3 \mathrm{~mm}$ thickness is shown in Figure 8 . The dark band is the residual material of a Kapton foil which was forgotten to remove. The 2 images are a comparison of a C-mode image of an air-coupled ultrasound scan (left) and a lock-in thermography phase image (right) taken with $0.3 \mathrm{~Hz}$. The frayed edges that are visible on the aircoupled ultrasound scan are caused by acoustic scattering signals and do not indicate any material defects. As the scale indicates, the colour shows the material's permeability for the ultrasound signal (134 kHz). Blue colour would indicate almost 
no permeability for the ultrasound signal which is typical for delaminated areas. With both methods the carbon fibre orientation and the band of residual Kapton foil is clearly detectable. However, the material's microstructure appears with slightly more contrast in the phase image of the thermographic analysis. The band-shaped defect is even better visible in its full extend (width) and some wave like structures of the carbon fabric near the edges of the plate are noticeable which cannot be detected with the air-coupled ultrasound technique. However, the main benefit of the lock-in thermography technique is the measurement time for this CFRP plate of approximately $320 \times 180 \mathrm{~mm}$. While for the ultrasound scan the measurement time was approximately 3 hours (with $1 \mathrm{~mm}$ width for each scanning line) it took only 3.5 minutes to obtain the lock-in thermography phase image.

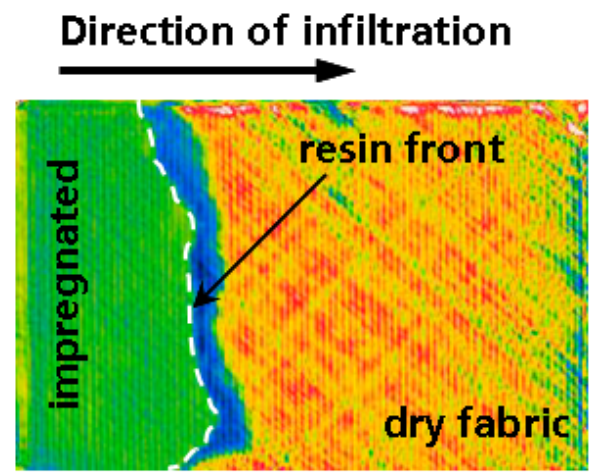

Excitation frequency: $0.3 \mathrm{~Hz}$

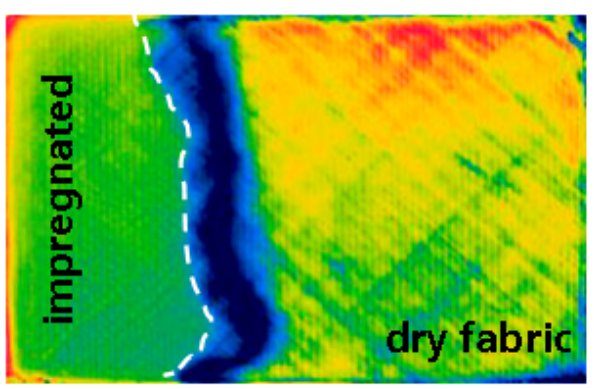

Excitation frequency: $0.06 \mathrm{~Hz}$

Fig. 7. Analysis of infiltration process of a carbon fibre pre-form at different excitation frequencies
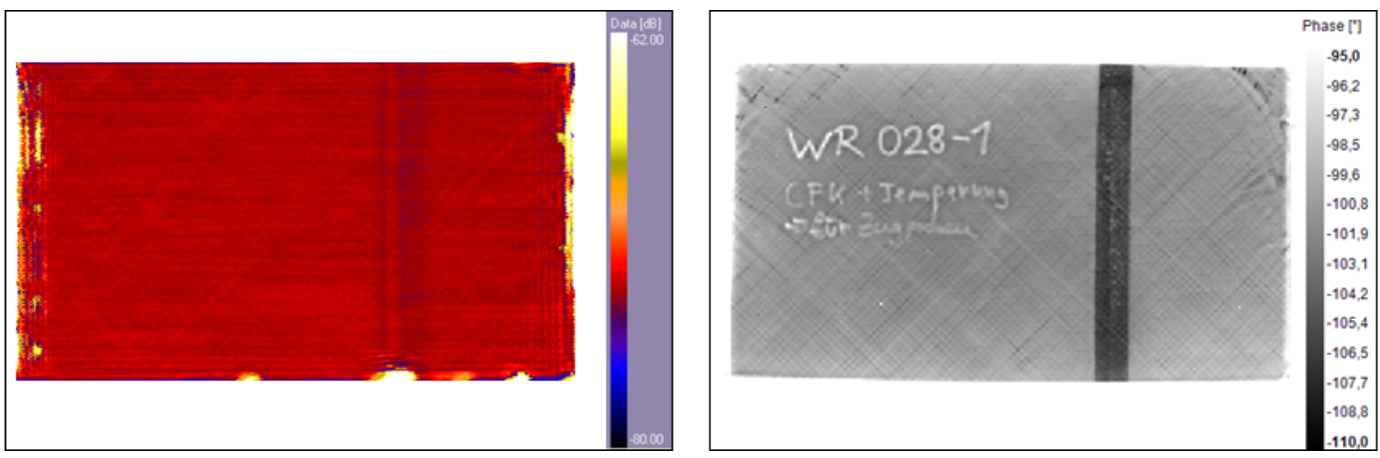

Fig. 8. A CFRP plate of a formerly wounded fibre layup. Comparison of ultrasound C-mode mapping (left) and lock-in thermography imaging (right)

For choosing a non-destructive test method for monitoring manufacturing steps within a process chain, a reliable detection of material defects and imaging of quality relevant structural features is essential. Even more important is the ability of widespread measurement on complex shaped and curved component surfaces combined with the shortest possible measurement time. Figure 9 shows another example of lock-in thermography application to focus on different material depths of an aircraft structure. The CFRP landing flap which is shown in all 4 images (A-D) is $900 \mathrm{~mm}$ long (wall thickness: $4 \mathrm{~mm}$ ) with decreasing excitation frequency from left to right $(A \Rightarrow D)$. Once more, it is obvious that with a decreasing excitation frequency the diffusion length of the thermal waves is increasing. High frequencies are the right choice for characterizing surface near features like the carbon fibre orientation (A). Slightly higher frequencies reveal defects such as a delamination that is caused by a locally cracked stringer (marked by a circle in image B). Still increasing the excitation frequency shows structural details of deeper regions such as the reinforcement elements (stringers). With a combined measurement sequence of at least 2 different excitation frequencies, it is also possible to measure the material's wall thickness according to the gray values of the phase images. 


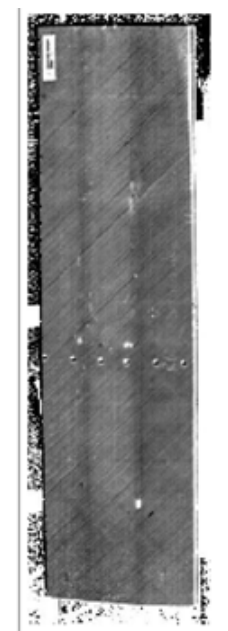

A

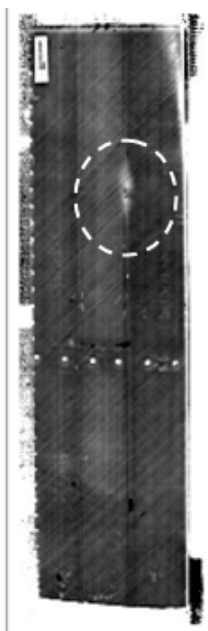

B

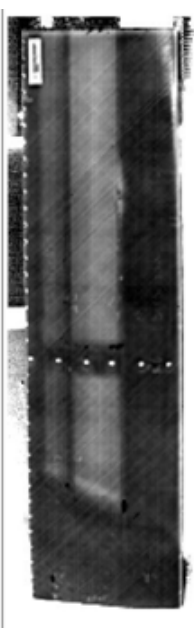

C

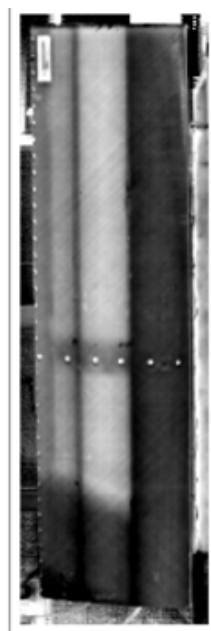

$\mathrm{D}$

Fig. 9. Cut out of an aircraft landing flap (length $900 \mathrm{~mm}$ ). Lock-in thermography analysis at different excitation frequencies (EDEVIS)

\section{Conclusions}

Fast and widespread applicable non-destructive measurement techniques are playing a key role in a process integrated quality assurance system for the manufacturing of large CFRP components. The limitation of detail resolution may be compensated by fast overview measurements combined with a fast data acquisition and full automated evaluation. The reliability of image data interpretation will be increased by implying additional data of reference measurements obtained from non-destructive testing with methods of much higher resolution as for example computed tomography or, if possible, with water-coupled ultrasound inspection. As demonstrated by several examples, lock-in thermography combines the benefits of a fast overview measurement with sufficient defect selectivity and high contrast imaging. Therefore, this test method will play an important role for process integrated non-destructive testing.

The non-destructive measuring head systems that will be used in a process chain must be adapted to the shape of the component and used on a large scaled in order to be able to inspect and assess the material properties of several square metres of large structural components in the shortest possible time. The new type of the test head should be specifically adapted for industrial use in the serial production of components and should interface with existing robot systems.

\section{REFERENCES}

[1] Ullmann T. and Schmidt T., "Quality assurance in the aircraft industry - nothing is left to chance," DLR Magazine 126.07, to be published, 2010.

[2] Busse G., Wu D. and Karpen W., "Thermal Wave Imaging with Phase Sensitive Modulated Thermography," J. Appl. Phys. 71, p. 3962-3965, 1992.

[3] Ullmann T., Jemmali R. and Hofmann S., "Non-Destructive Evaluation Methods for CMC Components," Proceedings ICACC-2009, Daytona Beach, FL (USA), available on CD, 2009.

[4] Chatzigeorgiou L., "Examination of Leakage Behaviour on Vacuum Infusion Processes," diploma thesis (in German), University of Stuttgart, 2005.

[5] Chatzigeorgiou L., Feiler M., Aoki R., „Feasibility Study for Process Monitoring on Vacuum Infiltration Processes with Lockin-Thermography,” (in German), German Soc. of Non-Destructive Testing, DGZfP Berichtsband 86-CD, p. 83-90, 2003.

[6] Wu D., Salerno A., Malter U., Aoki R., Kochendörfer R., Kächele P.K., Woithe K., Pfister K. and Busse G., "Inspection of Aircraft Structural Components using Lockin-Thermography," Proceedings QIRT'96, Edizioni ETS, pp. 251-256.

[7] Wu D., Wu C.Y. and Busse G., "Investigation in Resolution in Lock-in Thermography: Theory and Experiment," Proceedings QIRT'97, Edizioni ETS, pp. 269-274, 1997. 